\title{
Prong Analysis of Multiple Production of Pions at Bevatron Energy According to a Statistical Theory.
}

\author{
F. Gerbug and $I$. von Benr \\ CERN - Geneva \\ (Nuono ('imento, 16, $1046(1960))$
}

ADDENDA

The eaptions of the individual figures have unfortunately been omitied in the publication. 'They read as follows:

Fig. 1. - p-spectrum of 2 prong events.

Fig. 2. $-\pi^{\perp}$-spectrum of 2 prong events.

Fig. 3. - p-t-t-spectrum of 2 prong events.

Fig. 4. - p-spectrum of 4 prong events.

lig. 5. $-\pi$-spectrum of 4 prong events.

Fig. 6. $-p+\pi$-spectrum of 4 prong events.

Fig. 7. - p-speetrum of 6 prong events.

Fig. $8 .-\pi^{ \pm}$-spectrum of 6 prong events.

Fig. 9. $\cdots p+\pi^{ \pm}$-spectrum of 6 prong events.

Fig. 10. - p-spectrum of all events.

Fig. 11, ... $\pi$-spectrum of all events.

Hig. 12. $-\pi+p$-spectrum of all events. 